\title{
Infinite speed of support propagation for the Derrida-Lebowitz-Speer-Spohn equation and quantum drift-diffusion models
}

\author{
Julian Fischer
}

\begin{abstract}
We show that weak solutions of the Derrida-Lebowitz-SpeerSpohn (DLSS) equation display infinite speed of support propagation. We apply our method to the case of the quantum drift-diffusion equation which augments the DLSS equation with a drift term and possibly a second-order diffusion term. The proof is accomplished using weighted entropy estimates, Hardy's inequality and a family of singular weight functions to derive a differential inequality; the differential inequality shows exponential growth of the weighted entropy, with the growth constant blowing up very fast as the singularity of the weight becomes sharper. To the best of our knowledge, this is the first example of a nonnegativity-preserving higher-order parabolic equation displaying infinite speed of support propagation.
\end{abstract}

Mathematics Subject Classification (2010). 35K25, 35B05, 35K55, 35Q40, 82D37.

Keywords. Derrida-Lebowitz-Speer-Spohn equation; DLSS equation; Infinite speed of propagation; Qualitative behaviour; Higher-order parabolic equation.

\section{Introduction}

The Derrida-Lebowitz-Speer-Spohn equation

$$
u_{t}=-\nabla \cdot\left(u \nabla \frac{\Delta \sqrt{u}}{\sqrt{u}}\right)
$$

is of mathematical interest due to three reasons: first, as shown by Derrida et al. [8], its one-dimensional version arises in the study of interfaces in the Toom model, a stochastic cellular automaton describing the evolution of a spin lattice. Second, it is the equation associated with the leading order operator of 
quantum drift-diffusion models, i.e. drift-diffusion models for charge transport in semiconductor devices which include lowest-order quantum corrections; for a derivation of the quantum drift-diffusion equation, see the article by Degond et al. [7] and the references therein. Third, it is the equation associated with the gradient flow of the Fisher information $\int|\nabla \sqrt{u}|^{2} d x$ with respect to the Wasserstein distance as shown by Gianazza et al. [13].

Regarding existence of weak nonnegative solutions, for $d \leq 3$ and different boundary conditions a satisfactory theory of existence has been developed independently by Jüngel and Matthes [17] and by Gianazza et al. [13]; see also $[1,18,20]$ for other existence results. Recently, uniqueness of weak solutions in some class of sufficiently regular solutions has been shown by the author [10]. Note that the entropies found by Jüngel and Matthes [15] imply that the solutions of the DLSS equation constructed by them [17] belong to this class of uniqueness.

To the best of our knowledge, almost all results on qualitative behaviour of weak solutions to the DLSS equation are concerned with large time behaviour or perturbations of a steady state (see e.g. Jüngel and Toscani [19], Caceres et al. [2], Gualdani et al. [14], Dolbeault et al. [9]). Regarding positivity of solutions of the DLSS equation, only for $d=1$ it is known that (at least formally) $\int\left|\log u_{0}\right| d x<\infty$ implies $\int|\log u(., t)| d x<\infty$ for all $t>0$. However, to the best of our knowledge there are in particular no results on the short-time behaviour of the support in case $\operatorname{supp} u_{0} \neq \Omega$.

In the present work, we prove that weak solutions of the DLSS equation display infinite speed of support propagation; more precisely, we prove that for any nontrivial solution supp $u$ necessarily equals $\Omega \times[0, \infty)$ if $\Omega$ is connected.

Deriving lower bounds on the support of solutions to nonnegativitypreserving higher-order parabolic equations is a difficult task due to the lack of a comparison principle; to the best of the author's knowledge, no lower bounds on support propagation for higher-order nonnegativity-preserving parabolic equations have been available until recently, when the author managed to solve this problem in the case of the thin-film equation [11,12]. The author's method proceeds by looking for some weighted versions of backward entropies, which turn out to be strictly increasing with respect to time by themselves; then a differential inequality argument analogous to the one by Chipot and Sideris [6], who derived upper bounds on waiting times for the porous medium equation, applies. By these means the author has obtained upper bounds on waiting times for the thin-film equation as well as optimal lower bounds on asymptotic support propagation rates.

In the present work, we use a nontrivial adaption of this method. In the case of the one-dimensional DLSS equation with periodic boundary conditions, we roughly insert $|x|^{\gamma}$ (where $\gamma<0$ ) as a test function into the weak formulation of the equation and apply Hardy's inequality to deduce a differential inequality which shows that $\int u(., t)|x|^{\gamma} d x$ grows exponentially with time. The growth constant of the exponential function is seen to behave like $|\gamma|^{3}$; thus passing to the limit $\gamma \rightarrow-\infty$ we obtain a contradiction. In the multidimensional case, we need the reformulation of the DLSS equation in terms of 
$\sqrt{u}$ which the author has proven to hold for weak solutions within the class of uniqueness in [10]; we then again insert $|x|^{\gamma}$ and use Hardy's inequality to obtain a differential inequality, arguing analogously to the one-dimensional case. For the quantum drift-diffusion equation, we are faced with the problem of additional negative terms of the form $-|\gamma| b(t) \int \sqrt{u}(., t)|x|^{\gamma} d x$ appearing on the right-hand side of the differential inequality, where $b \in L_{l o c}^{p}([0, \infty))$ for some $p>1$. We show that the effect of these terms on the solutions of the differential inequality is negligible compared to the effect of the positive term $|\gamma|^{3} \int \sqrt{u}(., t)|x|^{\gamma} d x$.

Throughout the paper, we use standard notation for Sobolev spaces. By abuse of notation, by e.g. $L^{2}\left(I ; W_{l o c}^{1,2}(\Omega)\right)$ we denote the space of functions $u$ which for all $K \subset \subset \Omega$ satisfy $u \in L^{2}\left(I ; W^{1,2}(K)\right)$. We set $I:=[0, \infty)$. By $L_{l o c}^{2}(I ; X)$ we denote the set of all mappings $v: I \rightarrow X$ which belong to $L^{2}([0, T] ; X)$ for all $T>0$. By $\left(H^{2}\right)_{l o c}^{\prime}(\Omega)$ we denote the space of all distributions on $\Omega$ which belong to $H^{-2}(K)$ for all $K \subset \subset \Omega$.

\section{Main results}

Jüngel and Matthes [17] have introduced the following definition of weak solutions of the DLSS equation with periodic boundary conditions:

Definition 1. Suppose $\Omega=\left[S^{1}\right]^{d}$. Let $u_{0} \in L^{1}(\Omega)$ be given with $u_{0} \geq 0$. We call a nonnegative function $u \in L^{\infty}\left(I ; L^{1}(\Omega)\right)$ with $u \in W^{1,1}\left(I ; H^{-2}(\Omega)\right)$ and $\sqrt{u} \in L^{2}\left(I ; H^{2}(\Omega)\right)$ a weak solution to the DLSS equation with initial data $u_{0}$ if for all $\psi \in L^{\infty}\left(I ; H^{2}(\Omega)\right)$ we have

$$
\int_{0}^{T}\left\langle\partial_{t} u, \psi\right\rangle+\int_{0}^{T} \int_{\Omega}\left(\sqrt{u} D^{2} \sqrt{u}-\nabla \sqrt{u} \otimes \nabla \sqrt{u}\right): D^{2} \psi d x d t=0
$$

for all $T>0$ and if in addition $u(., 0)=u_{0}($.$) in H^{-2}(\Omega)$.

For $d \leq 3$, existence of weak solutions in case $u_{0} \log u_{0} \in L^{1}\left(\left[S^{1}\right]^{d}\right)$ has been shown in [17].

For our second result, we use the following weaker definition of local weak solution (where "local" refers to space, not time) which does not impose any boundary conditions:

Definition 2. Let $\Omega \subset \mathbb{R}^{d}$ be open or let $\Omega=\left[S^{1}\right]^{d}$. Let $u_{0} \in L^{1}(\Omega)$ be given with $u_{0} \geq 0$. We call a nonnegative measurable function $u$ with $u \in$ $W_{l o c}^{1,1}\left(I ;\left(H^{2}\right)_{l o c}^{\prime}(\Omega)\right)$ and $\sqrt{u} \in L_{l o c}^{2}\left(I ; H_{l o c}^{2}(\Omega)\right)$ a local weak solution to the DLSS equation with initial data $u_{0}$ if for all $\psi \in L^{\infty}\left(I ; H^{2}\right)$ with the property $\bigcup_{t \in I} \operatorname{supp} \psi(., t) \subset \subset \Omega$ we have

$$
\int_{0}^{T}\left\langle\partial_{t} u, \psi\right\rangle d t+\int_{0}^{T} \int_{\Omega}\left(\sqrt{u} D^{2} \sqrt{u}-\nabla \sqrt{u} \otimes \nabla \sqrt{u}\right): D^{2} \psi d x d t=0
$$

for all $T>0$ and if in addition $u(., 0)=u_{0}($.$) in \left(H^{2}\right)_{l o c}^{\prime}(\Omega)$.

Our third result involves quantum drift-diffusion models. As we are interested in qualitative behaviour of solutions, we only need to consider the case of 
a single charge-carrier species in an arbitrary but fixed electric field (subject to some regularity conditions). Our result then applies to all solutions of coupled quantum drift-diffusion models, as for a given solution the density of any fixed species solves a scalar QDD equation with given electric field. $u$ reads

The (dimensionless) quantum drift-diffusion equation for a single species

$$
u_{t}=-\nabla \cdot\left(u \nabla \frac{\Delta \sqrt{u}}{\sqrt{u}}\right)-\nabla \cdot(u \nabla V)+\nabla \cdot(\vartheta \nabla u),
$$

where $\vartheta>0$ denotes the temperature. Existence of solutions for such models has been shown e.g. by Chen et al. [5], Chen and Chen [4], and Chen and Ju [3]. We shall work with the following notion of local (with respect to space) weak solution:

Definition 3. Assume that $\Omega \subset \mathbb{R}^{d}$ is open or that $\Omega=\left[S^{1}\right]^{d}$, where $1 \leq d \leq 3$. Let $u_{0} \in L^{1}(\Omega)$ be given with $u_{0} \geq 0$. Let $V \in L_{l o c}^{\infty}\left(I ; L_{l o c}^{1}(\Omega)\right)$ be given with $\Delta V \in L_{l o c}^{\infty}\left(I ; L_{l o c}^{1}\right) \cap L_{l o c}^{1}(\Omega)\left(I ; H_{l o c}^{2}(\Omega)\right)$. Let $u$ be a nonnegative function with $u \in L_{l o c}^{\infty}\left(I ; L_{l o c}^{1}(\Omega)\right) \cap W_{l o c}^{1,1}\left(I ;\left(H^{2}\right)_{l o c}^{\prime}(\Omega)\right)$ and $\sqrt{u} \in L_{l o c}^{2}\left(I ; H_{l o c}^{2}(\Omega)\right)$.

We then call $u$ a local weak solution of the quantum drift-diffusion equation with initial data $u_{0}$ if for all $\psi \in L^{\infty}\left(I ; H^{2}\right)$ with $\bigcup_{t \in I} \operatorname{supp} \psi(., t) \subset \subset \Omega$ we have

$$
\begin{aligned}
\int_{0}^{T}\left\langle\partial_{t} u, \psi\right\rangle d t= & -\int_{0}^{T} \int_{\Omega}\left(\sqrt{u} D^{2} \sqrt{u}-\nabla \sqrt{u} \otimes \nabla \sqrt{u}\right): D^{2} \psi d x d t \\
& +\int_{0}^{T} \int_{\Omega} \vartheta \Delta u \psi+u \nabla V \cdot \nabla \psi d x d t
\end{aligned}
$$

for any $T>0$ and if in addition $u(., 0)=u_{0}($.$) in \left(H^{2}\right)_{l o c}^{\prime}(\Omega)$.

Again our definition does not include any boundary condition. Note that existence of such solutions has not yet been established; however, as formal calculations yield energy estimates which imply bounds for solutions in the stated norms, we expect such weak solutions to exist, at least for periodic boundary conditions (and presumably also for Dirichlet-Neumann boundary conditions).

Our first main result deals with the one-dimensional case. It reads as follows:

Theorem 4. Let $u$ be a weak solution of the DLSS equation on $S^{1}$. Suppose that $\left\|u_{0}\right\|_{L^{1}}>0$. We then have $\operatorname{supp} u=S^{1} \times[0, \infty)$.

In the case of several spatial dimensions, we need to impose an additional regularity assumption on the solution, namely $u^{\frac{1}{4}} \in L^{2}\left(I ; H^{2}\right)$. However, this regularity is implied by the entropy estimates for the DLSS equation and thus is satisfied for the solutions constructed by Jüngel and Matthes [17]. Moreover, note that this additional regularity is precisely the additional regularity of solutions within the class of uniqueness obtained by the author [10].

The second main result covers the case of several spatial dimensions. 
Theorem 5. Suppose that $\Omega \subset \subset \mathbb{R}^{d}$ is connected or that $\Omega=\left[S^{1}\right]^{d}$. Let $u$ be a local weak solution of the DLSS equation with the additional regularity $u^{\frac{1}{4}} \in L_{l o c}^{2}\left(I ; H_{l o c}^{2}(\Omega)\right)$. Assume that $\left\|u_{0}\right\|_{L^{1}}>0$ and that $u \in L_{l o c}^{\infty}\left(I ; L^{1}(\Omega)\right)$. Then for every $\delta>0$ and every $x_{0} \in \Omega$ we have supp $u \cap\left(B_{\delta}\left(x_{0}\right) \times[0, \delta)\right) \neq \emptyset$.

Remark 6. Note that in case of periodic or no-flux boundary conditions, mass is conserved and therefore the condition $u \in L_{l o c}^{\infty}\left(I ; L^{1}(\Omega)\right)$ is always satisfied. Moreover, in this case we have $\|u(., t)\|_{L^{1}}>0$ for every $t>0$ and therefore we obtain

$$
\operatorname{supp} u=\Omega \times[0, \infty)
$$

(since the theorem can be applied starting at any time $t_{0}>0$ instead of $t_{0}=0$ ).

Our third result covers the case of quantum drift-diffusion models:

Theorem 7. Suppose that $\Omega \subset \subset \mathbb{R}^{d}$ is connected or that $\Omega=\left[S^{1}\right]^{d}$. Let $u$ be a local weak solution of the quantum drift-diffusion equation with the additional regularity $u^{\frac{1}{4}} \in L_{l o c}^{2}\left(I ; H_{l o c}^{2}(\Omega)\right)$. Suppose that $\left\|u_{0}\right\|_{L^{1}}>0$. Then for every $\delta>0$ and every $x_{0} \in \Omega$ we have $\operatorname{supp} u \cap\left(B_{\delta}\left(x_{0}\right) \times[0, \delta)\right) \neq \emptyset$.

Again, the regularity assumption $u^{\frac{1}{4}} \in L_{l o c}^{2}\left(I ; H_{l o c}^{2}(\Omega)\right)$ follows from (formal) entropy estimates; thus, we expect weak solutions with the stated regularity to exist.

Note that again in case of periodic or no-flux boundary conditions, mass is conserved and therefore the condition $u \in L_{l o c}^{\infty}\left(I ; L^{1}\right)$ is always satisfied. Moreover, in this case we have $\|u(., t)\|_{L^{1}}>0$ for every $t>0$ and therefore we again obtain $\operatorname{supp} u=\Omega \times[0, \infty)$.

\section{Infinite speed of propagation for the DLSS equation}

We now proceed to the proof of infinite speed of support propagation for solutions of the DLSS equation. We first prove two Hardy-like inequalities which will be required.

Lemma 8. (Hardy-like inequalities) Let $\psi \in C^{\infty}\left(\mathbb{R}^{d} \backslash\{0\}\right)$ be given with $\Delta \psi>$ 0 on $\mathbb{R}^{d} \backslash\{0\}$. For any nonnegative $v \in H^{2}$ with $\operatorname{supp} v \subset \subset \mathbb{R}^{d} \backslash\{0\}$, the inequality

$$
\int v \Delta \psi d x \leq \int \frac{|\Delta v|^{2}}{v} \cdot \frac{|\psi|^{2}}{\Delta \psi} d x
$$

holds. Moreover, for any nonnegative $v \in H^{1}$ with $\operatorname{supp} v \subset \subset \mathbb{R}^{d} \backslash\{0\}$, the inequality

$$
\int v^{2} \Delta \psi d x \leq 4 \int|\nabla v|^{2} \cdot \frac{|\nabla \psi|^{2}}{\Delta \psi} d x
$$

is satisfied. 
Proof. Regarding the first assertion, for smooth compactly supported nonnegative $v$ we calculate

$$
\begin{aligned}
\int v \Delta \psi d x & =\int \Delta v \cdot \psi d x \\
& \leq\left(\int_{\operatorname{supp} v}(v+\epsilon) \Delta \psi d x\right)^{\frac{1}{2}}\left(\int_{\operatorname{supp} v} \frac{|\Delta v|^{2}}{v+\epsilon} \cdot \frac{|\psi|^{2}}{\Delta \psi} d x\right)^{\frac{1}{2}}
\end{aligned}
$$

By approximation (convolution of $v$ with a smoothing kernel and passing to the limit) this formula remains valid for any $v$ with the properties stated in the lemma. Passing to the limit $\epsilon \rightarrow 0$ using the monotone convergence theorem, we finish the proof.

Regarding the second assertion, for smooth compactly supported nonnegative $v$ we calculate

$$
\begin{aligned}
\int v^{2} \Delta \psi d x & =-2 \int v \nabla v \cdot \nabla \psi d x \\
& \leq 2\left(\int_{\operatorname{supp} v} v^{2} \Delta \psi d x\right)^{\frac{1}{2}}\left(\int_{\operatorname{supp} v}|\nabla v|^{2} \cdot \frac{|\nabla \psi|^{2}}{\Delta \psi} d x\right)^{\frac{1}{2}}
\end{aligned}
$$

By approximation (convolution of $v$ with a smoothing kernel and passing to the limit) this formula remains valid for any $v$ with the properties stated in the lemma.

We now prove our main result in the one-dimensional case with periodic boundary conditions.

Proof of Theorem 4. Let us rearrange the weak formulation of the DLSS equation (1) by integration by parts to yield

$$
\int_{0}^{T}\left\langle\partial_{t} u, \psi\right\rangle d t=2 \int_{0}^{T} \int_{S^{1}}\left|\sqrt{u}_{x}\right|^{2} \psi_{x x} d x d t-\frac{1}{2} \int_{0}^{T} \int_{S^{1}} u \psi_{x x x x} d x d t .
$$

We argue by contradiction. Suppose that there exist $x_{0} \in S^{1}, \delta>0,0<t_{S}<$ $t_{E}$ such that $\operatorname{supp} u \cap\left(B_{\delta}\left(x_{0}\right) \times\left[t_{S}, t_{E}\right)\right)=\emptyset$. We may identify $S^{1}$ with $[0,1)$. Without loss of generality, we may assume $x_{0}=0$. We plug in $x^{\gamma}$ as a test function in the weak formulation, where $\gamma<-4$. This is possible as by our assumption this test function is smooth in $\left.\operatorname{supp} u\right|_{S^{1} \times\left[t_{S}, t_{E}\right)}$. Thus we obtain for any $t_{0} \in\left(t_{S}, t_{E}\right)$ and any $T \in\left(t_{0}, t_{E}\right)$

$$
\begin{aligned}
\left.\int_{(0,1)} u(., t) x^{\gamma} d x\right|_{t_{0}} ^{T}= & 2 \gamma(\gamma-1) \int_{t_{0}}^{T} \int_{(0,1)}\left|\sqrt{u}_{x}\right|^{2} x^{\gamma-2} d x d t \\
& -\frac{1}{2} \gamma(\gamma-1)(\gamma-2)(\gamma-3) \int_{t_{0}}^{T} \int_{(0,1)} u(., t) x^{\gamma-4} d x d t .
\end{aligned}
$$

Recall that by Lemma 8 we have

$$
\int_{(0,1)} \sqrt{u}^{2} x^{\gamma-4} d x \leq \frac{4}{(\gamma-3)^{2}} \int_{(0,1)}\left|\sqrt{u}_{x}\right|^{2} x^{\gamma-2} d x
$$


Using (3) it follows that

$$
\begin{aligned}
\left.\int_{(0,1)} u(., t) x^{\gamma} d x\right|_{t_{0}} ^{T} \geq & \frac{1}{2} \gamma(\gamma-1)\left((\gamma-3)^{2}-(\gamma-2)(\gamma-3)\right) \\
& \cdot \int_{t_{0}}^{T} \int_{(0,1)} u(., t) x^{\gamma-4} d x d t \\
= & -\frac{1}{2} \gamma(\gamma-1)(\gamma-3) \int_{t_{0}}^{T} \int_{(0,1)} u(., t) x^{\gamma-4} d x d t \\
\geq & -\frac{1}{2} \gamma(\gamma-1)(\gamma-3) \int_{t_{0}}^{T} \int_{(0,1)} u(., t) x^{\gamma} d x d t
\end{aligned}
$$

where in the last step we have utilized $x^{-4} \geq 1$ on $(0,1)$. Gronwall's Lemma now implies that

$$
\begin{aligned}
\int_{(0,1)} u(., T) x^{\gamma} d x & \geq e^{-\frac{1}{2} \gamma(\gamma-1)(\gamma-3)\left(T-t_{0}\right)} \int_{(0,1)} u\left(., t_{0}\right) x^{\gamma} d x \\
& \geq e^{-\frac{1}{2} \gamma(\gamma-1)(\gamma-3)\left(T-t_{0}\right)}\left\|u_{0}\right\|_{L^{1}}
\end{aligned}
$$

where again we have used $x^{\gamma} \geq 1$ on $(0,1)$. We now note that

$$
\int_{(0,1)} u(., T) x^{\gamma} d x \leq \delta^{\gamma}\|u(., T)\|_{L^{1}}=\delta^{\gamma}\left\|u_{0}\right\|_{L^{1}}
$$

where we have used conservation of mass and the fact that $x^{\gamma} \leq \delta^{\gamma}$ on $\operatorname{supp} u(., t)$ for $t \in\left[t_{S}, t_{E}\right)$. Putting this estimate and the inequality (4) together, we obtain

$$
\delta^{\gamma}\left\|u_{0}\right\|_{L^{1}} \geq e^{-\frac{1}{2} \gamma(\gamma-1)(\gamma-3)\left(T-t_{0}\right)}\left\|u_{0}\right\|_{L^{1}}
$$

or equivalently in case $\left\|u_{0}\right\|_{L^{1}}>0$

$$
1 \geq e^{-\gamma\left[\log \delta+\frac{1}{2}(\gamma-1)(\gamma-3)\left(T-t_{0}\right)\right]}
$$

which yields a contradiction if we let $\gamma \rightarrow-\infty$ for $\delta>0$ and $T>t_{0}$ fixed. This is possible as $\gamma<-4$ is arbitrary. Thus our assertion is proved.

The following result has been established in [10] in case of weak solutions (see Lemma 15 in [10]); however, the proof for local weak solutions is entirely analogous.

Theorem 9. Given any local weak solution $u$ of the DLSS equation with the additional regularity $u^{\frac{1}{4}} \in L_{\text {loc }}^{2}\left(I ; H_{\text {loc }}^{2}\right)$, we have for any $\psi \in L^{\infty}\left(I ; W^{2, \infty}\right) \cap$ $W^{1,1}\left(I ; L^{\infty}\right)$ with $\psi(., T) \equiv 0$ and $\bigcup_{t \in[0, T]} \operatorname{supp} \psi(., t) \subset \subset \Omega$ 


$$
\begin{aligned}
& -2 \int_{0}^{T}\left\langle\sqrt{u}, \psi_{t}\right\rangle d t-2 \int \sqrt{u_{0}} \psi(., 0) d x \\
& =\int_{0}^{T} \int \frac{\psi}{\sqrt{u}}|\Delta \sqrt{u}|^{2} d x d t-\int_{0}^{T} \int \Delta \sqrt{u} \Delta \psi d x d t .
\end{aligned}
$$

By the following lemma (whose first part is well-known), the term $\frac{|\Delta \sqrt{u}|^{2}}{\sqrt{u}}$ is well-defined and belongs to $L_{l o c}^{1}\left(I ; L_{l o c}^{1}\right)$ :

Lemma 10. Let $d \leq 3$. Given $v \in H_{\text {loc }}^{2}(\Omega)$ with $v \geq 0$ and $\phi \in C_{c}^{\infty}(\Omega)$, we have $v^{\frac{1}{2}} \in W_{\text {loc }}^{1,4}(\Omega)$ with the estimate

$$
\int \phi^{4}\left|\nabla v^{\frac{1}{2}}\right|^{4} d x \leq C(d) \int \phi^{4}\left|D^{2} v\right|^{2} d x+C(d) \int v^{2}|\nabla \phi|^{4} d x .
$$

Moreover, for $v \in H_{l o c}^{2}(\Omega)$ with $v \geq 0$ and $\sqrt{v} \in H_{l o c}^{2}(\Omega)$ we have $\Delta v=0$ a.e. on $\{v=0\}$ and

$$
\frac{|\Delta v|^{2}}{v}=4 \chi_{v \neq 0} \cdot\left(\Delta \sqrt{v}+4\left|\nabla v^{\frac{1}{4}}\right|^{2}\right)^{2}
$$

pointwise a.e..

Proof. We calculate for smooth strictly positive $v$

$$
\begin{aligned}
\int\left|\partial_{i} v^{\frac{1}{2}}\right|^{4} \phi^{4} d x & =\frac{1}{16} \int v^{-2}\left|\partial_{i} v\right|^{4} \phi^{4} d x \\
& =\frac{3}{16} \int v^{-1}\left|\partial_{i} v\right|^{2} \partial_{i i}^{2} v \phi^{4} d x+\frac{1}{4} \int v^{-1}\left|\partial_{i} v\right|^{2} \partial_{i} v \partial_{i} \phi \phi^{3} d x .
\end{aligned}
$$

By Young's inequality we obtain

$$
\int\left|\partial_{i} v^{\frac{1}{2}}\right|^{4} \phi^{4} d x \leq C \int\left|\partial_{i i}^{2} v\right|^{2} \phi^{4} d x+C \int v^{2}\left|\partial_{i} \phi\right|^{4} d x .
$$

Taking the sum with respect to $i$, we obtain the first assertion of the lemma for smooth strictly positive $v$. By approximation the inequality carries over to the case of stricly positive $v \in H_{l o c}^{2}$. Considering $v+\epsilon$ and passing to the limit $\epsilon \rightarrow 0$, we finally see that the first assertion of the lemma holds for any nonnegative $v \in H_{l o c}^{2}$.

Regarding the second assertion of the lemma, we first note that the first assertion of the lemma applied to $\sqrt{v}$ yields $v^{\frac{1}{4}} \in W_{l o c}^{1,4}(\Omega)$. For smooth strictly positive $v$ we calculate

$$
\frac{|\Delta v|^{2}}{v}=4 \frac{|\nabla \cdot(\sqrt{v} \nabla \sqrt{v})|^{2}}{v}=4 \frac{\left(\sqrt{v} \Delta \sqrt{v}+|\nabla \sqrt{v}|^{2}\right)^{2}}{v}
$$

which implies

$$
\frac{|\Delta v|^{2}}{v}=4\left(\Delta \sqrt{v}+4\left|\nabla v^{\frac{1}{4}}\right|^{2}\right)^{2} .
$$

By approximation, the latter identity remains valid a.e. for strictly positive $v \in H_{l o c}^{2}$ (note that by $d \leq 3$ we have $v \in L_{l o c}^{\infty}$ ). To prove the identity for 
all nonnegative $v \in H_{l o c}^{2}$ with $\sqrt{v} \in H_{l o c}^{2}$, we consider $v+\epsilon$ in place of $v$ and deduce by the chain rule (note that $\Delta(v+\epsilon)=\Delta v$ )

$$
\frac{|\Delta v|^{2}}{v+\epsilon}=4\left(\frac{\sqrt{v}}{\sqrt{v+\epsilon}} \Delta \sqrt{v}+\frac{\epsilon}{(v+\epsilon)^{\frac{3}{2}}}|\nabla \sqrt{v}|^{2}+4 \frac{v^{\frac{3}{2}}}{(v+\epsilon)^{\frac{3}{2}}}\left|\nabla v^{\frac{1}{4}}\right|^{2}\right)^{2} .
$$

Passing to the limit $\epsilon \rightarrow 0$, the desired assertions are obtained as by dominated convergence the right-hand side converges strongly in $L_{l o c}^{1}$ to the desired limit (note that $\nabla \sqrt{v}=2 v^{\frac{1}{4}} \nabla v^{\frac{1}{4}}$ ) and the left-hand side converges pointwise a.e..

We are now in position to prove the infinite speed of propagation result in up to three spatial dimensions. We first show that the assertion of the theorem holds for any point $x_{0} \in \Omega$ with $\operatorname{dist}\left(x_{0}, \operatorname{supp} u_{0}\right)<\operatorname{dist}\left(x_{0}, \partial \Omega\right)$; the general case will be seen to follow below.

Lemma 11. Suppose that $\Omega \subset \subset \mathbb{R}^{d}$ is open or $\Omega=\left[S^{1}\right]^{d}$ with $d \leq 3$. Let $u$ be a local weak solution of the DLSS equation with the additional regularity $u^{\frac{1}{4}} \in L_{l o c}^{2}\left(I ; H_{l o c}^{2}(\Omega)\right)$. Assume that $u \in L_{l o c}^{\infty}\left(I ; L^{1}(\Omega)\right)$ and that $\operatorname{supp} u_{0} \neq \emptyset$.

Given $x_{0} \in \Omega$ with $\operatorname{dist}\left(x_{0}, \operatorname{supp} u_{0}\right)<\operatorname{dist}\left(x_{0}, \partial \Omega\right)$, for any $\delta>0$ we have $\operatorname{supp} u \cap\left(B_{\delta}\left(x_{0}\right) \times[0, \delta)\right) \neq \emptyset$.

Proof. We argue by contradiction. Assume that there exists $\delta>0$ such that $\left(B_{\delta}(x) \times[0, \delta)\right) \cap \operatorname{supp} u=\emptyset$. Set $r:=\operatorname{dist}\left(x_{0}, \operatorname{supp} u_{0}\right)$ and $R:=\operatorname{dist}\left(x_{0}, \partial \Omega\right)$; by assumption we have $r<R$. Take a smooth nonnegative cutoff $\phi$ with $\phi \equiv 1$ on $B_{\frac{2 r+R}{3}}\left(x_{0}\right)$ and $\phi \equiv 0$ on $\mathbb{R}^{d} \backslash B_{\frac{r+2 R}{3}}\left(x_{0}\right)$. Let $\xi \in C^{\infty}(\mathbb{R})$ be a smooth monotonous function with $\xi \equiv 1$ for $s<0$ and $\xi \equiv 0$ for $x>1$. Let $t_{0} \in(0, \delta)$ and $\gamma<-d-4$. We use $\psi(x, t):=\phi^{4}(x)\left|x-x_{0}\right|^{\gamma} \cdot \xi\left(\frac{t-t_{0}}{\epsilon}\right)$ as a test function in formula (5) (which is applicable by our assumptions).

Letting $\epsilon \rightarrow 0$ and noting that $\sqrt{u} \in W_{l o c}^{1,1}\left(I ;\left(H^{2}\right)_{l o c}^{\prime}\right) \cap L_{l o c}^{\infty}\left(I ; L_{l o c}^{2}\right)$ implies weak continuity in $L_{l o c}^{2}$ of the map $t \mapsto \sqrt{u(., t)}$, we obtain

$$
\begin{aligned}
& 2 \int \sqrt{u\left(., t_{0}\right)} \phi^{4}\left|x-x_{0}\right|^{\gamma} d x-2 \int \sqrt{u_{0}} \phi^{4}\left|x-x_{0}\right|^{\gamma} d x \\
& \quad=\int_{0}^{t_{0}} \int \frac{\phi^{4} \cdot\left|x-x_{0}\right|^{\gamma}}{\sqrt{u}}|\Delta \sqrt{u}|^{2} d x d t-\int_{0}^{t_{0}} \int \Delta \sqrt{u} \Delta\left(\phi^{4} \cdot\left|x-x_{0}\right|^{\gamma}\right) d x d t .
\end{aligned}
$$

Let $0 \leq t_{1}<t_{2}<\delta$. We obtain (since the previous formula holds for any $\left.t_{0} \in(0, \delta)\right)$

$$
\begin{aligned}
& 2 \int \sqrt{u\left(., t_{2}\right)} \phi^{4}\left|x-x_{0}\right|^{\gamma} d x=2 \int \sqrt{u\left(., t_{1}\right)} \phi^{4}\left|x-x_{0}\right|^{\gamma} d x \\
& \quad+\int_{t_{1}}^{t_{2}} \int \frac{\left|x-x_{0}\right|^{\gamma}}{\phi^{4} \sqrt{u}}\left|\Delta\left[\phi^{4} \sqrt{u}\right]\right|^{2} d x d t-\int_{t_{1}}^{t_{2}} \int \phi^{4} \sqrt{u} \Delta^{2}\left|x-x_{0}\right|^{\gamma} d x d t
\end{aligned}
$$




$$
\begin{aligned}
& +\int_{t_{1}}^{t_{2}} \int \frac{\phi^{4}\left|x-x_{0}\right|^{\gamma}}{\sqrt{u}}|\Delta \sqrt{u}|^{2}-\frac{\left|x-x_{0}\right|^{\gamma}}{\phi^{4} \sqrt{u}}\left|\Delta\left[\phi^{4} \sqrt{u}\right]\right|^{2} d x d t \\
& -\int_{t_{1}}^{t_{2}} \int \sqrt{u} \Delta^{2}\left[\phi^{4}\left|x-x_{0}\right|^{\gamma}\right]-\phi^{4} \sqrt{u} \Delta^{2}\left|x-x_{0}\right|^{\gamma} d x d t .
\end{aligned}
$$

Note that $\sqrt{\phi^{4} \sqrt{u}}=\phi^{2} u^{\frac{1}{4}} \in L_{\text {loc }}^{2}\left(I ; H_{l o c}^{2}\right)$; thus we know by Lemma 10 that the expression $\frac{1}{\sqrt{\phi^{4} \sqrt{u}}} \Delta\left[\phi^{4} \sqrt{u}\right]$ is well-defined and belongs to $L_{l o c}^{2}\left(I ; L_{l o c}^{2}\right)$.

Lemma 8 applied to $v:=\phi^{4} \sqrt{u}$ and $\psi:=\Delta\left|x-x_{0}\right|^{\gamma}$ for $\gamma<1-d$ states that

$$
\frac{(\gamma-2)(\gamma-4+d)}{\gamma(\gamma-2+d)} \int \phi^{4} \sqrt{u} \Delta^{2}\left|x-x_{0}\right|^{\gamma} d x \leq \int \frac{\left|\Delta\left[\phi^{4} \sqrt{u}\right]\right|^{2}}{\phi^{4} \sqrt{u}}\left|x-x_{0}\right|^{\gamma} d x .
$$

Using this inequality in (6), we get

$$
\begin{aligned}
& 2 \int \sqrt{u\left(., t_{2}\right)} \phi^{4}\left|x-x_{0}\right|^{\gamma} d x \geq 2 \int \sqrt{u\left(., t_{1}\right)} \phi^{4}\left|x-x_{0}\right|^{\gamma} d x \\
& +(\gamma-2)(\gamma-4+d)(-4 \gamma-2 d+8) \int_{t_{1}}^{t_{2}} \int \phi^{4} \sqrt{u}\left|x-x_{0}\right|^{\gamma-4} d x d t \\
& \quad+\int_{t_{1}}^{t_{2}} \int \frac{\phi^{4}\left|x-x_{0}\right|^{\gamma}}{\sqrt{u}}|\Delta \sqrt{u}|^{2}-\frac{\left|x-x_{0}\right|^{\gamma}}{\phi^{4} \sqrt{u}}\left|\Delta\left[\phi^{4} \sqrt{u}\right]\right|^{2} d x d t \\
& -\int_{t_{1}}^{t_{2}} \int \sqrt{u} \Delta^{2}\left[\phi^{4}\left|x-x_{0}\right|^{\gamma}\right]-\phi^{4} \sqrt{u} \Delta^{2}\left|x-x_{0}\right|^{\gamma} d x d t .
\end{aligned}
$$

Note that the difference in the latter two integrals is nonzero only on supp $\nabla \phi$, i.e. it is zero outside of $B_{\frac{r+2 R}{3}}\left(x_{0}\right) \backslash B_{\frac{2 r+R}{3}}\left(x_{0}\right)$. Moreover we have

$$
\begin{aligned}
& \left\|\frac{1}{\phi^{4} \sqrt{u}}\left|\Delta\left[\phi^{4} \sqrt{u}\right]\right|^{2}\right\|_{L^{1}\left(B_{\frac{r+2 R}{3}}\left(x_{0}\right)\right)}+\left\|\frac{1}{\sqrt{u}}|\Delta \sqrt{u}|^{2}\right\|_{L^{1}\left(B_{\frac{r+2 R}{3}}\left(x_{0}\right)\right)} \\
& \leq C(r, R)\left\|\phi^{2} u^{\frac{1}{4}}\right\|_{H^{2}\left(B_{\frac{r+3 R}{4}}\left(x_{0}\right)\right)}^{2}+C(r, R)\left\|u^{\frac{1}{4}}\right\|_{H^{2}}^{2}\left(B_{\left.\frac{r+3 R}{4}\left(x_{0}\right)\right)}\right. \\
& \leq C(r, R)\left\|u^{\frac{1}{4}}\right\|_{H^{2}\left(B_{\frac{r+3 R}{4}}\left(x_{0}\right)\right)}^{2}
\end{aligned}
$$

(the first inequality is a consequence of the second assertion of Lemma 10 applied to $v:=\phi^{4} \sqrt{u}$ and $v:=\sqrt{u}$ using some suitable cutoff $\tilde{\phi}$; the second inequality follows noting that $\phi$ depends only on $r$ and $R$ ). Note that

$$
\left|\Delta\left(\phi^{4} \sqrt{u}\right)-\phi^{4} \Delta \sqrt{u}\right| \leq C \phi^{3}|\nabla \phi| u^{\frac{1}{4}}\left|\nabla u^{\frac{1}{4}}\right|+C \phi^{2}|\nabla \phi|^{2} \sqrt{u}+C \phi^{3}|\Delta \phi| \sqrt{u}
$$

which implies

$$
\begin{aligned}
& \left|\frac{1}{\phi^{2} u^{\frac{1}{4}}} \Delta\left(\phi^{4} \sqrt{u}\right)-\frac{\phi^{2}}{u^{\frac{1}{4}}} \Delta \sqrt{u}\right| \\
& \quad \leq C(r, R) \chi_{\left\{x: \frac{2 r+R}{3} \leq\left|x-x_{0}\right| \leq \frac{r+2 R}{3}\right\}} \cdot\left(u^{\frac{1}{4}}+\left|\nabla u^{\frac{1}{4}}\right|\right) .
\end{aligned}
$$


Moreover, we have

$$
\begin{aligned}
& \left|\phi^{4} \Delta^{2}\right| x-\left.x_{0}\right|^{\gamma}-\Delta^{2}\left(\phi^{4}\left|x-x_{0}\right|^{\gamma}\right) \mid \\
& \leq C(r, R) \chi_{\left\{x: \frac{2 r+R}{3} \leq\left|x-x_{0}\right| \leq \frac{r+2 R}{3}\right\}} \cdot\left(\left|x-x_{0}\right|^{\gamma}+|\gamma| \cdot\left|x-x_{0}\right|^{\gamma-1}\right. \\
& \left.\quad+\left(|\gamma|^{2}+1\right) \cdot\left|x-x_{0}\right|^{\gamma-2}+\left(|\gamma|^{3}+1\right) \cdot\left|x-x_{0}\right|^{\gamma-3}\right) .
\end{aligned}
$$

Putting (8)-(10) together, we therefore obtain (note that the constant does not depend on $\gamma$ )

$$
\begin{aligned}
& \left.\left|\int_{t_{1}}^{t_{2}} \int \frac{\phi^{4}\left|x-x_{0}\right|^{\gamma}}{\sqrt{u}}\right| \Delta \sqrt{u}\right|^{2}-\frac{\left|x-x_{0}\right|^{\gamma}}{\phi^{4} \sqrt{u}}\left|\Delta\left[\phi^{4} \sqrt{u}\right]\right|^{2} d x d t \mid \\
& \quad+\left|\int_{t_{1}}^{t_{2}} \int \sqrt{u} \Delta^{2}\left[\phi^{4}\left|x-x_{0}\right|^{\gamma}\right]-\left[\phi^{4} \sqrt{u}\right] \Delta^{2}\right| x-\left.x_{0}\right|^{\gamma} d x d t \mid \\
& \leq C(r, R)\left(1+|\gamma|^{3}\right) \int_{t_{1}}^{t_{2}}\left[\frac{2 r+R}{3}\right]^{\gamma}|| u^{\frac{1}{4}}||_{H^{2}\left(B_{\frac{r+3 R}{4}}\left(x_{0}\right)\right)}^{2} d t .
\end{aligned}
$$

Using this inequality in (7), this proves that

$$
\begin{aligned}
\int \sqrt{u\left(., t_{2}\right)} \phi^{4}\left|x-x_{0}\right|^{\gamma} d x \geq \int \sqrt{u\left(., t_{1}\right)} \phi^{4}\left|x-x_{0}\right|^{\gamma} d x \\
\quad+\frac{1}{2}(\gamma-2)(\gamma-4+d)(-4 \gamma-2 d+8) \int_{t_{1}}^{t_{2}} \int \phi^{4} \sqrt{u}\left|x-x_{0}\right|^{\gamma-4} d x d t \\
\quad-\left.C(r, R)\left(1+|\gamma|^{3}\right)\left[\frac{2 r+R}{3}\right]^{\gamma} \int_{t_{1}}^{t_{2}}|| u^{\frac{1}{4}}\right|_{H^{2}\left(B_{\frac{r+3 R}{4}}\left(x_{0}\right)\right)} ^{2} d t \\
\geq \quad \int \sqrt{u\left(., t_{1}\right)} \phi^{4}\left|x-x_{0}\right|^{\gamma} d x \\
\quad+\frac{1}{2}(\gamma-2)(\gamma-4+d)(-4 \gamma-2 d+8) R^{-4} \int_{t_{1}}^{t_{2}} \int \phi^{4} \sqrt{u}\left|x-x_{0}\right|^{\gamma} d x d t \\
\quad-C(r, R)\left(1+|\gamma|^{3}\right)\left[\frac{2 r+R}{3}\right]^{\gamma} \int_{t_{1}}^{t_{2}}\left\|u^{\frac{1}{4}}\right\|_{H^{2}\left(B_{\frac{r+3 R}{4}}\left(x_{0}\right)\right)}^{2} d t .
\end{aligned}
$$

Knowing that $r=\operatorname{dist}\left(x_{0}, \operatorname{supp} u_{0}\right)$ we see that $M_{0}:=\int_{B_{\frac{3 r+R}{4}}\left(x_{0}\right)} \sqrt{u_{0}} d x>0$ and therefore

$$
\int \sqrt{u(., 0)} \phi^{4}\left|x-x_{0}\right|^{\gamma} d x \geq\left[\frac{3 r+R}{4}\right]^{\gamma} M_{0}>0 .
$$

Here we have used that $\phi \equiv 1$ on $B_{\frac{2 r+R}{3}}\left(x_{0}\right)$. We now apply Gronwall's inequality to (11) and our assumption $\left(B_{\delta}\left(x_{0}\right) \times[0, \delta)\right) \cap \operatorname{supp} u=\emptyset$ to yield for 
$t \in(0, \delta)$

$$
\begin{aligned}
\delta^{\gamma} \int \sqrt{u(., t)} \phi^{4} d x \geq & \int \sqrt{u(., t)} \phi^{4}\left|x-x_{0}\right|^{\gamma} d x \geq\left(\int \sqrt{u(., 0)} \phi^{4}\left|x-x_{0}\right|^{\gamma} d x\right. \\
& \left.-C(r, R)\left(1+|\gamma|^{3}\right)\left[\frac{2 r+R}{3}\right]^{\gamma} \int_{0}^{t} \|\left. u^{\frac{1}{4}}\right|_{\left.H^{2}\left(B_{\frac{r+3 R}{4}}\left(x_{0}\right)\right) d t\right)} ^{2} d t\right) \\
& \cdot \exp \left(\frac{1}{2} R^{-4}(\gamma-2)(\gamma-4+d)(-4 \gamma-2 d+8) t\right) \\
\geq & \left(\left[\frac{3 r+R}{4}\right]^{\gamma} M_{0}-C(r, R)\left(1+|\gamma|^{3}\right)\left[\frac{2 r+R}{3}\right]^{\gamma}\right. \\
& \times \int_{0}^{\delta}\left\|u^{\frac{1}{4}}\right\|_{H^{2}}^{2}\left(B_{\left.\frac{r+3 R}{4}\left(x_{0}\right)\right)} d t\right) \\
& \cdot \exp \left(\frac{1}{2} R^{-4}(\gamma-2)(\gamma-4+d)(-4 \gamma-2 d+8) t\right) .
\end{aligned}
$$

For $-\gamma$ large enough (depending on $r, R, M_{0}$, and $u$ ), the first factor can be estimated from below by $\frac{1}{2}\left[\frac{3 r+R}{4}\right]^{\gamma} M_{0}$ since $\frac{3 r+R}{4}<\frac{2 r+R}{3}$. Dividing both sides of the inequality by $\delta^{\gamma}$ we obtain for $t \in(0, \delta)$

$$
\begin{aligned}
\int \sqrt{u(., t)} \phi^{4} d x \geq & \frac{1}{2} M_{0} \exp \left(\frac{1}{2} R^{-4}(\gamma-2)(\gamma-4+d)(-4 \gamma-2 d+8) t\right. \\
& \left.+\gamma \log \frac{3 r+R}{4}-\gamma \log \delta\right) .
\end{aligned}
$$

Letting $\gamma \rightarrow-\infty$ we get $\int \sqrt{u(., t)} \phi^{4} d x=\infty$ for any $t \in(0, \delta)$. With $\phi$ being compactly supported, this contradicts the fact that $u \in L_{l o c}^{\infty}\left(I ; L_{l o c}^{1}\right)$ and therefore finishes the proof.

Proof of Theorem 5. Having shown the assertion of the theorem for any $x_{0} \in \Omega$ with $\operatorname{dist}\left(x_{0}, \operatorname{supp} u_{0}\right)<\operatorname{dist}\left(x_{0}, \partial \Omega\right)$, the general case follows easily by iterating the result of the lemma: Given an arbitrary point $x_{0} \in \Omega$ and a point $y \in \Omega$ with $\operatorname{dist}\left(y, \operatorname{supp} u_{0}\right)<\operatorname{dist}(y, \partial \Omega)$ (such a point exists as otherwise $\left.\operatorname{supp} u_{0}=\emptyset\right)$, there exists a path $\alpha:[0,1] \rightarrow \Omega$ with $\alpha(0)=y$ and $\alpha(1)=x_{0}$. By compactness of the interval $[0,1]$ and continuity of $\alpha$, for any $n$ sufficiently large we have

$$
\operatorname{dist}\left(\alpha\left(\frac{k}{n}\right), \alpha\left(\frac{k+1}{n}\right)\right)+\frac{1}{n}<\operatorname{dist}(\alpha([0,1]), \partial \Omega)
$$

for all $k \in\{0, \ldots, n-1\}$. We additionally require $n \geq \frac{1}{\delta}$.

Since $\operatorname{dist}\left(y, \operatorname{supp} u_{0}\right)<\operatorname{dist}(y, \partial \Omega)$, by the previous lemma there exists $t_{0} \in\left[0, \frac{\delta}{n+1}\right)$ such that the inequality $\operatorname{dist}\left(\alpha(0), \operatorname{supp} u\left(., t_{0}\right)\right)<\frac{1}{n}$ holds (note that by $u \in W_{l o c}^{1,1}\left(I ;\left(H^{2}\right)_{l o c}^{\prime}\right)$ and by $\sup _{t \in[0, \delta)}\|u(., t)\|_{L^{1}}<\infty$, we know that the mapping $t \mapsto u(., t)$ is a weak-* continuous mapping with values in the 
space of Radon measures). This implies

$$
\begin{aligned}
& \operatorname{dist}\left(\alpha\left(\frac{1}{n}\right), \operatorname{supp} u\left(., t_{0}\right)\right) \\
& \leq \operatorname{dist}\left(\alpha\left(\frac{0}{n}\right), \alpha\left(\frac{1}{n}\right)\right)+\operatorname{dist}\left(\alpha\left(\frac{0}{n}\right), \operatorname{supp} u\left(., t_{0}\right)\right) \\
& \quad \leq \operatorname{dist}\left(\alpha\left(\frac{0}{n}\right), \alpha\left(\frac{1}{n}\right)\right)+\frac{1}{n} \\
& \quad<\operatorname{dist}(\alpha([0,1]), \partial \Omega) \\
& \leq \operatorname{dist}\left(\alpha\left(\frac{1}{n}\right), \partial \Omega\right) .
\end{aligned}
$$

We can therefore apply the previous lemma again (starting at time $t_{0}$ instead of 0$)$ to obtain $t_{1} \in\left[t_{0}, \frac{2 \delta}{n+1}\right)$ satisfying

$$
\operatorname{dist}\left(\alpha\left(\frac{1}{n}\right), \operatorname{supp} u\left(., t_{1}\right)\right)<\frac{1}{n} .
$$

More generally, let $k \in\{1, \ldots, n-1\}$ and assume that there exists $t_{k} \in$ $\left[0, \frac{\delta(k+1)}{n+1}\right)$ such that

$$
\operatorname{dist}\left(\alpha\left(\frac{k}{n}\right), \operatorname{supp} u\left(., t_{k}\right)\right)<\frac{1}{n} .
$$

Then we deduce that

$$
\begin{aligned}
& \operatorname{dist}\left(\alpha\left(\frac{k+1}{n}\right), \operatorname{supp} u\left(., t_{k}\right)\right) \\
& \leq \operatorname{dist}\left(\alpha\left(\frac{k+1}{n}\right), \alpha\left(\frac{k}{n}\right)\right)+\operatorname{dist}\left(\alpha\left(\frac{k}{n}\right), \operatorname{supp} u\left(., t_{k}\right)\right) \\
& \leq \operatorname{dist}\left(\alpha\left(\frac{k+1}{n}\right), \alpha\left(\frac{k}{n}\right)\right)+\frac{1}{n} \\
& <\operatorname{dist}(\alpha([0,1]), \partial \Omega) \\
& \leq \operatorname{dist}\left(\alpha\left(\frac{k+1}{n}\right), \partial \Omega\right) .
\end{aligned}
$$

Thus, applying the previous lemma again with initial time $t_{k}$ instead of 0 , we see that there exists $t_{k+1} \in\left[t_{k}, t_{k}+\frac{\delta}{n}\right.$ ) (in particular $t_{k+1} \in\left[0, \frac{\delta(k+2)}{n+1}\right)$ ) with

$$
\operatorname{dist}\left(\alpha\left(\frac{k+1}{n}\right), \operatorname{supp} u\left(., t_{k+1}\right)\right)<\frac{1}{n} \text {. }
$$

Arguing by induction, we finally obtain a time $t_{n} \in[0, \delta)$ for which the estimate $\operatorname{dist}\left(\alpha(1), \operatorname{supp} u\left(., t_{n}\right)\right)<\frac{1}{n} \leq \delta$ is satisfied (recall that $\left.n \geq \frac{1}{\delta}\right)$. This finishes the proof since $\alpha(1)=x_{0}$. 


\section{Infinite speed of propagation for quantum drift-diffusion models}

We now adapt our method to the case of quantum drift-diffusion models. We need the following theorem which is proven by proceeding as in the proof of Theorem 9 in [10] (the proof in [10] is stated only for weak solutions, but it also applies without any changes to local weak solutions):

Theorem 12. Let $u$ be a local weak solution of the quantum drift-diffusion equation with the additional regularity $u^{\frac{1}{4}} \in L_{l o c}^{2}\left(I ; H_{l o c}^{2}\right)$. Then it holds that $\sqrt{u} \in W_{l o c}^{1,1}\left(I ;\left(H^{2}\right)_{l o c}^{\prime}\right) ;$ moreover, for any $\psi \in L^{\infty}\left(I ; H^{2}\right)$ with the property $\bigcup_{t \in I} \operatorname{supp} \psi(., t) \subset \subset \Omega$ we have

$$
\begin{aligned}
2 \int_{t_{1}}^{t_{2}}\left\langle\sqrt{u}_{t}, \psi\right\rangle d t= & \int_{t_{1}}^{t_{2}} \int_{\Omega} \frac{|\Delta \sqrt{u}|^{2}}{\sqrt{u}} \psi d x d t-\int_{t_{1}}^{t_{2}} \int_{\Omega} \Delta \sqrt{u} \Delta \psi d x d t \\
& +8 \vartheta \int_{t_{1}}^{t_{2}} \int_{\Omega}\left|\nabla u^{\frac{1}{4}}\right|^{2} \psi d x d t+2 \vartheta \int_{t_{1}}^{t_{2}} \int_{\Omega} \sqrt{u} \Delta \psi d x d t \\
& -\int_{t_{1}}^{t_{2}} \int_{\Omega} \psi \nabla V \cdot \nabla \sqrt{u} d x d t+\int_{t_{1}}^{t_{2}} \int_{\Omega} \sqrt{u} \nabla V \cdot \nabla \psi d x d t
\end{aligned}
$$

for a.e. $0<t_{1}<t_{2}$ and a.e. $t_{2}>0$ in case $t_{1}=0$.

Furthermore, the following Gronwall-type estimate will be required:

Lemma 13. (Gronwall's Lemma) Let $f:[0, T] \rightarrow \mathbb{R}$ be a nonnegative measureable function satisfying the integral inequality

$$
f(t) \geq f(0)+\int_{0}^{t}-b(t) f(t)-d(t)+e(t) d t,
$$

where $b, d, e \in L^{1}([0, T])$ are nonnegative. Then the estimate

$$
f(t) \geq\left(f(0)+\int_{0}^{t} e(t) d t\right) \cdot \exp \left(-\int_{0}^{t} b(t) d t\right)-\int_{0}^{T} d(t) d t
$$

holds true for all $t \in[0, T]$.

Proof. Define

$$
g(t):=\left(f(0)+\int_{0}^{t} e(t) d t\right) \cdot \exp \left(-\int_{0}^{t} b(t) d t\right)-\int_{0}^{T} d(t) d t .
$$

Writing $g(t)=g(0)+\int_{0}^{t} \frac{d}{d t} g(t) d t$ and using the integral inequality for $f$, by standard estimates we deduce

$$
f(t)-g(t) \geq-\int_{0}^{t} b(t)[f(t)-g(t)] d t .
$$


Reformulating this inequality in terms of $g-f$, we may apply Gronwall's inequality in its standard formulation to conclude.

The first step in the proof of Theorem 7 is the derivation of an estimate for our usual weighted entropy.

Lemma 14. Let $d \leq 3$. Assume that $u$ is a local weak solution to the quantum drift-diffusion equation with nonnegative initial data $u_{0}$. Suppose that $u^{\frac{1}{4}} \in$ $L_{\text {loc }}^{2}\left(I ; H_{l o c}^{2}(\Omega)\right)$. Let $x_{0} \in \Omega$. Set $R:=\operatorname{dist}\left(x_{0}, \partial \Omega\right)$ and $r:=\operatorname{dist}\left(x_{0}, \operatorname{supp} u_{0}\right)$. Assume that $0<r<R$ and let $\phi$ be a smooth function on $\Omega$ with $\operatorname{supp} \phi \subset$ $B_{\frac{r+2 R}{3}}\left(x_{0}\right)$ and $\phi \equiv 1$ on $B_{\frac{2 r+R}{3}}\left(x_{0}\right)$. Suppose that there exists $\delta>0$ with supp $u \cap\left(B_{\delta}\left(x_{0}\right) \times[0, \delta)\right)=\emptyset$. Then we have for any $\gamma<-d$ and any $0 \leq t_{1}<$ $t_{2}<\delta$

$$
\begin{aligned}
& \left.\int \sqrt{u}(., t)\left|x-x_{0}\right|^{\gamma} \phi^{4} d x\right|_{t_{1}} ^{t_{2}} \geq \frac{1}{2}(\gamma-2)(\gamma-4+d)(-4 \gamma-2 d+8) R^{-4} \\
& \quad \times \int_{t_{1}}^{t_{2}} \int \phi^{4} \sqrt{u}(., t)\left|x-x_{0}\right|^{\gamma} d x d t \\
& -\int_{t_{1}}^{t_{2}} \| \Delta V(., t)||_{L^{\infty}\left(B_{\tilde{R}}\left(x_{0}\right)\right)} \int_{\Omega} \sqrt{u}(., t)\left|x-x_{0}\right|^{\gamma} \phi^{4} d x d t \\
& -|\gamma| \int_{t_{1}}^{t_{2}} \frac{1}{\delta} \| \nabla V(., t)\left|L_{L^{\infty}\left(B_{\tilde{R}}\left(x_{0}\right)\right)} \int_{\Omega} \sqrt{u}(., t)\right| x-\left.x_{0}\right|^{\gamma} \phi^{4} d x d t \\
& -C(r, R, \phi)\left(1+|\gamma|^{3}\right)\left[\frac{2 r+R}{3}\right]^{\gamma} \int_{t_{1}}^{t_{2}}\left\|u^{\frac{1}{4}}\right\|_{H^{2}\left(B_{\tilde{R}}\left(x_{0}\right)\right)}^{2} d t \\
& -\left.C(r, R, \phi)\left[\frac{2 r+R}{3}\right]^{\gamma} \int_{t_{1}}^{t_{2}}\|\sqrt{u}(., t)\|\right|_{L^{2}\left(B_{\tilde{R}}\left(x_{0}\right)\right)}\|\nabla V(., t)\|_{L^{2}\left(B_{\tilde{R}}\left(x_{0}\right)\right)} d t
\end{aligned}
$$

where we have set $\tilde{R}:=\frac{r+3 R}{4}$.

Proof. We plug in $\left|x-x_{0}\right|^{\gamma} \phi^{4}$ as a test function in Theorem 12. This is possible since by our assumption, $\left|x-x_{0}\right|^{\gamma} \phi^{4}$ coincides on some neighbourhood of $\bigcup_{t \in[0, \delta)} \operatorname{supp} u(., t)$ with a smooth compactly supported function. Estimating the terms originating from the DLSS operator as in the proof of Lemma 11, we obtain for a.e. $0<t_{1}<t_{2}<\delta$ and a.e. $t_{2} \in(0, \delta)$ in case $t_{1}=0$

$$
\begin{aligned}
& 2 \int_{t_{1}}^{t_{2}}\left\langle\sqrt{u}_{t},\left|x-x_{0}\right|^{\gamma} \phi^{4}\right\rangle d t \geq(\gamma-2)(\gamma-4+d)(-4 \gamma-2 d+8) \\
& \quad \times \int_{t_{1}}^{t_{2}} \int \phi^{4} \sqrt{u}(., t)\left|x-x_{0}\right|^{\gamma-4} d x d t \\
& -C(r, R, \phi)\left(1+|\gamma|^{3}\right)\left[\frac{2 r+R}{3}\right]^{\gamma} \int_{t_{1}}^{t_{2}} \|\left. u^{\frac{1}{4}}\right|_{H^{2}\left(B_{\frac{r+3 R}{4}}\left(x_{0}\right)\right)} ^{2} d t \\
& +8 \vartheta \int_{t_{1}}^{t_{2}} \int_{\Omega}\left|\nabla u^{\frac{1}{4}}\right|^{2} \phi^{4}\left|x-x_{0}\right|^{\gamma} d x d t
\end{aligned}
$$




$$
\begin{aligned}
& +2 \vartheta \int_{t_{1}}^{t_{2}} \int_{\Omega} \sqrt{u} \Delta\left(\phi^{4}\left|x-x_{0}\right|^{\gamma}\right) d x d t \\
& -\int_{t_{1}}^{t_{2}} \int_{\Omega} \phi^{4}\left|x-x_{0}\right|^{\gamma} \nabla V \cdot \nabla \sqrt{u} d x d t \\
& +\int_{t_{1}}^{t_{2}} \int_{\Omega} \sqrt{u} \nabla V \cdot \nabla\left(\phi^{4}\left|x-x_{0}\right|^{\gamma}\right) d x d t .
\end{aligned}
$$

Since we have $\sqrt{u} \in W_{l o c}^{1,1}\left(I ;\left(H^{2}\right)_{l o c}^{\prime}\right)$ which implies that $\sqrt{u} \in C_{l o c}^{0}\left(I ;\left(H^{2}\right)_{l o c}^{\prime}\right)$ and since $\left|x-x_{0}\right|^{\gamma} \phi^{4}$ is smooth in some neighbourhood of $\bigcup_{t \in[0, \delta)} \operatorname{supp} u(., t)$, we in fact obtain this inequality for every $t_{1}, t_{2}$ with the stated restrictions (not just a.e. $t_{1}, t_{2}$ ).

Integrating by parts in the penultimate term and estimating, we obtain

$$
\begin{aligned}
2 & \left.\int \sqrt{u}(., t)\left|x-x_{0}\right|^{\gamma} \phi^{4} d x\right|_{t_{1}} ^{t_{2}} \geq(\gamma-2)(\gamma-4+d)(-4 \gamma-2 d+8) \\
& \times \int_{t_{1}}^{t_{2}} \int \phi^{4} \sqrt{u}(., t)\left|x-x_{0}\right|^{\gamma-4} d x d t \\
& +2 \vartheta \int_{t_{1}}^{t_{2}} \int_{\Omega} \sqrt{u} \phi^{4} \Delta\left|x-x_{0}\right|^{\gamma} d x d t \\
& -C \int_{t_{1}}^{t_{2}} \int_{\Omega} \sqrt{u} \phi^{3}|\nabla \phi| \cdot\left|x-x_{0}\right|^{\gamma-1} d x d t \\
& -C \int_{t_{1}}^{t_{2}} \int_{\Omega} \sqrt{u} \phi^{2}|\nabla \phi|^{2}\left|x-x_{0}\right|^{\gamma} d x d t \\
& -C \int_{t_{1}}^{t_{2}} \int_{\Omega} \sqrt{u} \phi^{3}|\Delta \phi| \cdot\left|x-x_{0}\right|^{\gamma} d x d t \\
& +\int_{t_{1}}^{t_{2}} \int_{\Omega} \phi^{4}\left|x-x_{0}\right|^{\gamma} \sqrt{u} \Delta V d x d t \\
& +2 \int_{t_{1}}^{t_{2}} \int_{\Omega} \sqrt{u} \nabla V \cdot \nabla\left(\phi^{4}\left|x-x_{0}\right|^{\gamma}\right) d x d t \\
& -C(r, R, \phi)\left(1+|\gamma|^{3}\right)\left[\frac{2 r+R}{3}\right]^{\gamma} \int_{t_{1}}^{t_{2}} \|\left. u^{\frac{1}{4}}\right|_{H^{2}\left(B_{\frac{r+3 R}{4}}\left(x_{0}\right)\right)} ^{2} d t .
\end{aligned}
$$

Using the fact that $\gamma(\gamma-2+d)>0$, we may drop the second term on the right-hand side. Using the fact that $\left|x-x_{0}\right| \geq \frac{2 r+R}{3}$ on supp $\nabla \phi$ and the boundedness of the first and the second derivative of $\phi$, we may estimate the third, fourth, and fifth term on the right-hand side to yield

$$
\begin{aligned}
& \left.2 \int \sqrt{u}(., t)\left|x-x_{0}\right|^{\gamma} \phi^{4} d x\right|_{t_{1}} ^{t_{2}} \\
& \quad \geq(\gamma-2)(\gamma-4+d)(-4 \gamma-2 d+8) \int_{t_{1}}^{t_{2}} \int \phi^{4} \sqrt{u}(., t)\left|x-x_{0}\right|^{\gamma-4} d x d t
\end{aligned}
$$




$$
\begin{aligned}
& +\int_{t_{1}}^{t_{2}} \int_{\Omega} \phi^{4}\left|x-x_{0}\right|^{\gamma} \sqrt{u} \Delta V d x d t \\
& +2 \gamma \int_{t_{1}}^{t_{2}} \int_{\Omega} \sqrt{u} \nabla V \cdot \phi^{4}\left|x-x_{0}\right|^{\gamma-2}\left(x-x_{0}\right) d x d t \\
& +8 \int_{t_{1}}^{t_{2}} \int_{\Omega} \sqrt{u} \nabla V \cdot \phi^{3}\left|x-x_{0}\right|^{\gamma} \nabla \phi d x d t \\
& -C(r, R, \phi)\left(1+|\gamma|^{3}\right)\left[\frac{2 r+R}{3}\right]^{\gamma} \int_{t_{1}}^{t_{2}}\left\|u^{\frac{1}{4}}\right\|_{H^{2}\left(B_{\frac{r+3 R}{4}}\left(x_{0}\right)\right)}^{2} d t .
\end{aligned}
$$

We abbreviate $\tilde{R}:=\frac{r+3 R}{4}$. Using again the fact that $\left|x-x_{0}\right| \geq \frac{2 r+R}{3}$ on $\operatorname{supp} \nabla \phi$ and in addition the fact that $\operatorname{supp} u(., t) \cap B_{\delta}\left(x_{0}\right)=\emptyset$ for $0 \leq t<\delta$, we obtain the desired inequality by estimating the second, the third, and the fourth term on the right-hand side.

Lemma 15. Let $d \leq 3$. Assume that $u$ is a local weak solution to the quantum drift-diffusion equation with nonnegative initial data $u_{0}$. Suppose that $u^{\frac{1}{4}} \in$ $L_{\text {loc }}^{2}\left(I ; H_{\text {loc }}^{2}(\Omega)\right)$. Let $x_{0} \in \Omega$. Set $R:=\operatorname{dist}\left(x_{0}, \partial \Omega\right)$ and $r:=\operatorname{dist}\left(x_{0}, \operatorname{supp} u_{0}\right)$. Assume that $0<r<R$ and let $\phi$ be a smooth function on $\Omega$ with $\operatorname{supp} \phi \subset$ $B_{\frac{r+2 R}{3}}\left(x_{0}\right)$ and $\phi \equiv 1$ on $B_{\frac{2 r+R}{3}}\left(x_{0}\right)$. Suppose that there exists $\delta>0$ with $\operatorname{supp} u \cap\left(B_{\delta}\left(x_{0}\right) \times[0, \delta)\right)=\emptyset$. Then there exists $\gamma_{0}<-d$ depending only on $r$, $R, \phi, u_{0}, \vartheta$ and $V$ such that for all $\gamma<\gamma_{0}$ the following assertion holds: For all $t_{0} \in[0, \delta)$ with

$$
\int \phi^{4} \sqrt{u\left(x, t_{0}\right)}\left|x-x_{0}\right|^{\gamma} d x \geq \int \phi^{4} \sqrt{u_{0}(x)}\left|x-x_{0}\right|^{\gamma} d x
$$

the estimate

$$
\int \phi^{4} \sqrt{u(x, t)}\left|x-x_{0}\right|^{\gamma} d x \geq \frac{1}{2} \int \phi^{4} \sqrt{u\left(x, t_{0}\right)}\left|x-x_{0}\right|^{\gamma} d x
$$

is satisfied for all $t \in\left(t_{0}, \min \left(t_{0}+\tau, \delta\right)\right)$, where $\tau:=|\gamma|^{-\frac{29}{10}}$.

Proof. By our assumptions, Lemma 14 is applicable. The Gagliardo-Nirenberg-Sobolev interpolation inequality implies that

$\|v\|_{L^{\infty}\left(B_{\tilde{R}}\left(x_{0}\right)\right)} \leq C(d, \tilde{R})\left\|D^{2} v\right\|_{L^{2}\left(B_{\tilde{R}}\left(x_{0}\right)\right)}^{\theta}\|v\|_{L^{1}\left(B_{\tilde{R}}\left(x_{0}\right)\right)}^{1-\theta}+C(d, \tilde{R})\|v\|_{L^{1}\left(B_{\tilde{R}}\left(x_{0}\right)\right)}$

holds with $\theta=\frac{2 d}{4+d}$. Knowing that $\Delta V \in L_{l o c}^{1}\left(I ; H_{l o c}^{2}\right) \cap L_{l o c}^{\infty}\left(I ; L_{l o c}^{1}\right)$, we therefore obtain $\Delta V \in L_{l o c}^{\frac{4+d}{2 d}}\left(I ; L^{\infty}\left(B_{\tilde{R}}\left(x_{0}\right)\right)\right)$. The GNS interpolation inequality also implies that

$$
\|v\|_{L^{4}\left(B_{\rho}\left(x_{0}\right)\right)} \leq C(d, \tilde{R})\left\|D^{2} v\right\|_{L^{2}\left(B_{\rho}\left(x_{0}\right)\right)}^{\theta}\|v\|_{L^{1}\left(B_{\rho}\left(x_{0}\right)\right)}^{1-\theta}+C(d, \tilde{R})\|v\|_{L^{1}\left(B_{\rho}\left(x_{0}\right)\right)}
$$

with $\theta=\frac{3 d}{8+2 d}$. We thus have $\Delta V \in L_{l o c}^{\frac{8+2 d}{3 d}}\left(I ; L^{4}\left(B_{\rho}\left(x_{0}\right)\right)\right)$ with $\rho:=\frac{r+4 R}{5}$ and therefore obtain by elliptic regularity theory and the Sobolev embedding (note 
that $d<4$ and $\tilde{R}<\rho)$ that $\nabla V \in L_{l o c}^{\frac{8+2 d}{3 d}}\left(I ; L^{\infty}\left(B_{\tilde{R}}\left(x_{0}\right)\right)\right)$. Since $\gamma<-d$ we infer from inequality (12) that

$$
\begin{aligned}
& \left.\int \sqrt{u}(., t)\left|x-x_{0}\right|^{\gamma} \phi^{4} d x\right|_{t_{1}} ^{t_{2}} \geq-\int_{t_{1}}^{t_{2}}\|\Delta V(., t)\|_{L^{\infty}\left(B_{\tilde{R}}\left(x_{0}\right)\right)} \int_{\Omega} \sqrt{u}(., t)\left|x-x_{0}\right|^{\gamma} \phi^{4} d x d t \\
& -|\gamma| \int_{t_{1}}^{t_{2}} \frac{1}{\delta}|| \nabla V(., t) \|_{L^{\infty}\left(B_{\tilde{R}}\left(x_{0}\right)\right)} \int_{\Omega} \sqrt{u}(., t)\left|x-x_{0}\right|^{\gamma} \phi^{4} d x d t \\
& -C(r, R, \phi)\left(1+|\gamma|^{3}\right)\left[\frac{2 r+R}{3}\right]^{\gamma} \int_{t_{1}}^{t_{2}}\left\|u^{\frac{1}{4}}(., t)\right\|_{H^{2}\left(B_{\tilde{R}}\left(x_{0}\right)\right)}^{2} d t \\
& -C(r, R, \phi)\left[\frac{2 r+R}{3}\right]^{\gamma} \int_{t_{1}}^{t_{2}}\|\sqrt{u}(., t)\|_{L^{2}\left(B_{\tilde{R}}\left(x_{0}\right)\right)}\|\nabla V(., t)\|_{L^{2}\left(B_{\tilde{R}}\left(x_{0}\right)\right)} d t .
\end{aligned}
$$

Recall that $\tau=|\gamma|^{-\frac{29}{10}}$. We then obtain by Lemma 13 for $t>t_{0}$

$$
\begin{aligned}
\int & \sqrt{u}(., t)\left|x-x_{0}\right|^{\gamma} \phi^{4} d x \\
\geq & \int \sqrt{u}\left(., t_{0}\right)\left|x-x_{0}\right|^{\gamma} \phi^{4} d x \\
& \cdot \exp \left(-\int_{t_{0}}^{t} \|\left.\Delta V(., \tilde{t})\right|_{L^{\infty}\left(B_{\tilde{R}}\left(x_{0}\right)\right)} d \tilde{t}\right. \\
& \left.-\frac{|\gamma|}{\delta} \int_{t_{0}}^{t}\|\nabla V(., \tilde{t})\|_{L^{\infty}\left(B_{\tilde{R}}\left(x_{0}\right)\right)} d \tilde{t}\right) \\
& -C(r, R, \phi)\left(1+|\gamma|^{3}\right)\left[\frac{2 r+R}{3}\right]^{\gamma} \int_{t_{0}}^{t}\left\|u^{\frac{1}{4}}(., \tilde{t})\right\|_{H^{2}\left(B_{\tilde{R}}\left(x_{0}\right)\right)}^{2} d \tilde{t} \\
& -C(r, R, \phi)\left[\frac{2 r+R}{3}\right]^{\gamma} \int_{t_{0}}^{t}\|\sqrt{u}(., \tilde{t})\|_{L^{2}\left(B_{\tilde{R}}\left(x_{0}\right)\right)}\|\nabla V(., \tilde{t})\|_{L^{2}\left(B_{\tilde{R}}\left(x_{0}\right)\right)} d \tilde{t} .
\end{aligned}
$$

Assuming that $t \in\left(t_{0}, \min \left(t_{0}+\tau, \delta\right)\right)$, we infer

$$
\begin{aligned}
\int & \sqrt{u}(., t)\left|x-x_{0}\right|^{\gamma} \phi^{4} d x \\
\geq & \int \sqrt{u}\left(., t_{0}\right)\left|x-x_{0}\right|^{\gamma} \phi^{4} d x \\
& \cdot \exp \left(-|| \Delta V(., t) \|_{L^{\frac{4+d}{2 d}}\left([0, \delta] ; L^{\infty}\left(B_{\tilde{R}}\left(x_{0}\right)\right)\right)} \tau^{\frac{4-d}{4+d}}\right. \\
& \left.-\frac{|\gamma|}{\delta}\|\nabla V(., t)\|_{L^{\frac{8+2 d}{3 d}}\left([0, \delta] ; L^{\infty}\left(B_{\tilde{R}}\left(x_{0}\right)\right)\right)} \tau^{\frac{8-d}{8+2 d}}\right)
\end{aligned}
$$




$$
\begin{aligned}
& -C(r, R, \phi)\left(1+|\gamma|^{3}\right)\left[\frac{2 r+R}{3}\right]^{\gamma} \int_{t_{0}}^{t}\left\|u^{\frac{1}{4}}(., \tilde{t})\right\|_{H^{2}\left(B_{\tilde{R}}\left(x_{0}\right)\right)}^{2} d \tilde{t} \\
& -C(r, R, \phi)\left[\frac{2 r+R}{3}\right]^{\gamma} \int_{t_{0}}^{t}\|\sqrt{u}(., \tilde{t})\|_{L^{2}\left(B_{\tilde{R}}\left(x_{0}\right)\right)}\|\nabla V(., \tilde{t})\|_{L^{2}\left(B_{\tilde{R}}\left(x_{0}\right)\right)} d \tilde{t}
\end{aligned}
$$

where we have used Hölder's inequality and the fact that $\left|t-t_{0}\right| \leq \tau$.

Recall that $\frac{8-d}{8+2 d} \geq \frac{5}{14}$ since $d \leq 3$; thus $|\gamma| \cdot \tau^{\frac{8-d}{8+2 d}} \leq|\gamma|^{1-\frac{145}{140}}$ (since $\gamma<-1$ and $\tau=|\gamma|^{-\frac{29}{10}}$ ). Thus there exists some $\gamma_{1}<-1$ such that for all $\gamma<\gamma_{1}$ we have

$$
\begin{aligned}
\frac{1}{4} \geq & \|\Delta V(., t)\|_{L^{\frac{4+d}{2 d}}\left([0, \delta] ; L^{\infty}\left(B_{\tilde{R}}\left(x_{0}\right)\right)\right)} \tau^{\frac{4-d}{4+d}} \\
& +\frac{|\gamma|}{\delta}\|\nabla V(., t)\|_{L^{\frac{8+2 d}{3 d}}\left([0, \delta] ; L^{\infty}\left(B_{\tilde{R}}\left(x_{0}\right)\right)\right)} \tau^{\frac{8-d}{8+2 d}} .
\end{aligned}
$$

Define

$$
M_{0}:=\int_{B_{\frac{3 r+R}{4}}\left(x_{0}\right)} \sqrt{u}(., 0) d x .
$$

Note that $M_{0}>0$ since $r=\operatorname{dist}\left(x_{0}, \operatorname{supp} u_{0}\right)$. There exists $\gamma_{2}<0$ such that

$$
\begin{aligned}
& C(r, R, \phi)\left(1+|\gamma|^{3}\right)\left[\frac{2 r+R}{3}\right]^{\gamma} \int_{0}^{\delta}\left\|u^{\frac{1}{4}}(., \tilde{t})\right\|_{H^{2}\left(B_{\tilde{R}}\left(x_{0}\right)\right)}^{2} d \tilde{t} \\
& \quad+C(r, R, \phi)\left[\frac{2 r+R}{3}\right]^{\gamma} \int_{0}^{\delta}\|\sqrt{u}(., \tilde{t})\|_{L^{2}\left(B_{\tilde{R}}\left(x_{0}\right)\right)}\|\nabla V(., \tilde{t})\|_{L^{2}\left(B_{\tilde{R}}\left(x_{0}\right)\right)} d \tilde{t} \\
& \leq \frac{1}{4}\left[\frac{3 r+R}{4}\right]^{\gamma} M_{0}
\end{aligned}
$$

is satisfied for all $\gamma<\gamma_{2}$ (as one easily checks, such a $\gamma_{2}$ exists since $r<R$ ).

We now define $\gamma_{0}:=\min \left(\gamma_{1}, \gamma_{2}\right)$. Since $\gamma<\min \left(\gamma_{1}, \gamma_{2}\right)$ holds, we deduce by inserting (15) and (17) in estimate (14) that

$$
\begin{aligned}
\int \sqrt{u}(., t)\left|x-x_{0}\right|^{\gamma} \phi^{4} d x \geq & \exp \left(-\frac{1}{4}\right) \cdot \int \sqrt{u}\left(., t_{0}\right)\left|x-x_{0}\right|^{\gamma} \phi^{4} d x \\
& -\frac{1}{4}\left[\frac{3 r+R}{4}\right]^{\gamma} M_{0} \geq \frac{1}{2} \int \sqrt{u}\left(., t_{0}\right)\left|x-x_{0}\right|^{\gamma} \phi^{4} d x,
\end{aligned}
$$

where in the last step we have used the bound $\exp \left(-\frac{1}{4}\right) \geq \frac{3}{4}$, assumption (13), the definition (16), as well as the fact that $\phi \equiv 1$ on $\frac{B_{\frac{3 r+R}{4}}}{4}\left(x_{0}\right)$.

Lemma 16. Let $d \leq 3$. Assume that $u$ is a local weak solution to the quantum drift-diffusion equation with nonnegative initial data $u_{0}$. Suppose that $u^{\frac{1}{4}} \in$ $L_{\text {loc }}^{2}\left(I ; H_{\text {loc }}^{2}(\Omega)\right)$. Let $x_{0} \in \Omega$. Set $R:=\operatorname{dist}\left(x_{0}, \partial \Omega\right)$ and $r:=\operatorname{dist}\left(x_{0}, \operatorname{supp} u_{0}\right)$. Assume that $0<r<R$ and let $\phi$ be a smooth function on $\Omega$ with $\operatorname{supp} \phi \subset$ $B_{\frac{r+2 R}{3}}\left(x_{0}\right)$ and $\phi \equiv 1$ on $B_{\frac{2 r+R}{3}}\left(x_{0}\right)$. Suppose that there exists $\delta>0$ with $\operatorname{supp} u \cap\left(B_{\delta}\left(x_{0}\right) \times[0, \delta)\right)=\emptyset^{3}$. Then there exists $\gamma_{0}<0$ depending only on 
$r, R, \phi, u_{0}, \vartheta$ and $V$ such that for all $\gamma<\gamma_{0}$ and $\tau:=|\gamma|^{-\frac{29}{10}}$ the following assertion holds: For all $t_{0} \in[0, \delta-\tau)$ with

$$
\int \phi^{4} \sqrt{u\left(., t_{0}\right)}\left|x-x_{0}\right|^{\gamma} d x \geq \int \phi^{4} \sqrt{u_{0}(.)}\left|x-x_{0}\right|^{\gamma} d x
$$

the estimate

$$
\begin{array}{r}
\int \phi^{4} \sqrt{u\left(., t_{0}+\tau\right)}\left|x-x_{0}\right|^{\gamma} d x \geq \frac{1}{8} \tau(\gamma-2)(\gamma-4+d)(-4 \gamma-2 d+8) \\
R^{-4} \int \phi^{4} \sqrt{u\left(., t_{0}\right)}\left|x-x_{0}\right|^{\gamma}
\end{array}
$$

is satisfied.

Proof. The proof is somewhat analogous to the proof of the previous lemma. We shall use the same choice of $\gamma_{0}, \gamma_{1}, \gamma_{2}$ as in the proof of the previous lemma. By our assumptions, Lemmas 14 and 15 are applicable. Let $t_{0} \leq t_{1}<$ $t_{2} \leq t_{0}+\tau$. Inserting the estimate from Lemma 15 in (12), we obtain

$$
\begin{aligned}
\int & \left.\sqrt{u}(., t)\left|x-x_{0}\right|^{\gamma} \phi^{4} d x\right|_{t_{1}} ^{t_{2}} \\
\geq & \frac{1}{4}(\gamma-2)(\gamma-4+d)(-4 \gamma-2 d+8) R^{-4} \int_{t_{1}}^{t_{2}} \int \phi^{4} \sqrt{u}\left(., t_{0}\right)\left|x-x_{0}\right|^{\gamma} d x d t \\
& -\int_{t_{1}}^{t_{2}}\|\Delta V(., t)\|_{L^{\infty}\left(B_{\tilde{R}}\left(x_{0}\right)\right)} \int_{\Omega} \sqrt{u}(., t)\left|x-x_{0}\right|^{\gamma} \phi^{4} d x d t \\
& -|\gamma| \int_{t_{1}}^{t_{2}} \frac{1}{\delta}\|\nabla V(., t)\| L_{L^{\infty}\left(B_{\tilde{R}}\left(x_{0}\right)\right)} \int_{\Omega} \sqrt{u}(., t)\left|x-x_{0}\right|^{\gamma} \phi^{4} d x d t \\
& -C(r, R, \phi)\left(1+|\gamma|^{3}\right)\left[\frac{2 r+R}{3}\right]^{\gamma} \int_{t_{1}}^{t_{2}}\left\|u^{\frac{1}{4}}\right\|_{H^{2}\left(B_{\tilde{R}}\left(x_{0}\right)\right)}^{2} d t \\
& -C(r, R, \phi)\left[\frac{2 r+R}{3}\right]^{\gamma} \int_{t_{1}}^{t_{2}}\|\sqrt{u}(., t)\|_{L^{2}\left(B_{\tilde{R}}\left(x_{0}\right)\right)}\|\nabla V(., t)\|_{L^{2}\left(B_{\tilde{R}}\left(x_{0}\right)\right)} d t .
\end{aligned}
$$

Applying Lemma 13, we get

$$
\begin{aligned}
\int & \sqrt{u}\left(., t_{0}+\tau\right)\left|x-x_{0}\right|^{\gamma} \phi^{4} d x \\
\geq & \left(1+\frac{1}{4} R^{-4}(\gamma-2)(\gamma-4+d)(-4 \gamma-2 d+8) \tau\right) \int \sqrt{u}\left(., t_{0}\right)\left|x-x_{0}\right|^{\gamma} \phi^{4} d x \\
& \cdot \exp \left(-\int_{t_{0}}^{t_{0}+\tau}\|\Delta V(., t)\|_{L^{\infty}\left(B_{\tilde{R}}\left(x_{0}\right)\right)} d t\right. \\
& \left.-\frac{|\gamma|}{\delta} \int_{t_{0}}^{t_{0}+\tau}\|\nabla V(., t)\|_{L^{\infty}\left(B_{\tilde{R}}\left(x_{0}\right)\right)} d t\right) \\
& -C(r, R, \phi)(1+|\gamma|)^{3}\left[\frac{2 r+R}{3}\right]^{\gamma} \int_{t_{0}}^{t_{0}+\tau}\left\|u^{\frac{1}{4}}(., t)\right\|_{H^{2}\left(B_{\tilde{R}}\left(x_{0}\right)\right)}^{2} d t
\end{aligned}
$$




$$
\begin{aligned}
& -C(r, R, \phi)\left[\frac{2 r+R}{3}\right]^{\gamma} \int_{t_{0}}^{t_{0}+\tau}\|\sqrt{u}(., t)\|_{L^{2}\left(B_{\tilde{R}}\left(x_{0}\right)\right)}\|\nabla V(., t)\|_{L^{2}\left(B_{\tilde{R}}\left(x_{0}\right)\right)} d t \\
\geq & \left(1+\frac{1}{4} R^{-4}(\gamma-2)(\gamma-4+d)(-4 \gamma-2 d+8) \tau\right) \int \sqrt{u}\left(., t_{0}\right)\left|x-x_{0}\right|^{\gamma} \phi^{4} d x \\
& \cdot \exp \left(-\|\Delta V(., t)\|_{L^{\frac{4+d}{2 d}}\left([0, \delta] ; L^{\infty}\left(B_{\tilde{R}}\left(x_{0}\right)\right)\right)} \tau^{\frac{4-d}{4+d}}\right. \\
& \left.-\frac{|\gamma|}{\delta}\|\nabla V(., t)\|_{L^{\frac{8+2 d}{3 d}}\left([0, \delta] ; L^{\infty}\left(B_{\tilde{R}}\left(x_{0}\right)\right)\right)} \tau^{\frac{8-d}{8+2 d}}\right) \\
& -C(r, R, \phi)(1+|\gamma|)^{3}\left[\frac{2 r+R}{3}\right]^{\gamma} \int_{t_{0}}^{t_{0}+\tau}\left\|u^{\frac{1}{4}}(., t)\right\|_{H^{2}\left(B_{\tilde{R}}\left(x_{0}\right)\right)}^{2} d t \\
& -C(r, R, \phi)\left[\frac{2 r+R}{3}\right]^{\gamma} \int_{t_{0}}^{t_{0}+\tau}\|\sqrt{u}(., t)\|_{L^{2}\left(B_{\tilde{R}}\left(x_{0}\right)\right)}\|\nabla V(., t)\|_{L^{2}\left(B_{\tilde{R}}\left(x_{0}\right)\right)} d t
\end{aligned}
$$

where we have used Hölder's inequality in the second step. Since we have $\gamma<\min \left(\gamma_{1}, \gamma_{2}\right)=\gamma_{0}$, the exponential term is bounded from below by $\frac{3}{4}$ (see (15)). By our assumption (18), the bound (17), the definition (16), and the fact that $\phi \equiv 1$ on $B_{\frac{3 r+R}{4}}\left(x_{0}\right)$, the inhomogeneities are bounded by $\frac{1}{4} \int \sqrt{u}\left(., t_{0}\right) \mid x-$ $\left.x_{0}\right|^{\gamma} \phi^{4} d x$. We therefore obtain

$$
\begin{aligned}
\int & \sqrt{u}\left(., t_{0}+\tau\right)\left|x-x_{0}\right|^{\gamma} \phi^{4} d x \\
\geq & {\left[\frac{3}{4}\left(1+\frac{1}{4} \tau(\gamma-2)(\gamma-4+d)(-4 \gamma-2 d+8) R^{-4}\right)-\frac{1}{4}\right] } \\
& \cdot \int \sqrt{u}\left(., t_{0}\right)\left|x-x_{0}\right|^{\gamma} \phi^{4} d x .
\end{aligned}
$$

This finishes the proof of the lemma.

Proof of Theorem \%. Again we only need to prove the assertion in case the condition $\operatorname{dist}\left(x_{0}, \operatorname{supp} u_{0}\right)<\operatorname{dist}\left(x_{0}, \partial \Omega\right)$ is satisfied, since the general case follows from this case as in the proof of Theorem 5 . Set $R:=\operatorname{dist}\left(x_{0}, \partial \Omega\right)$ and $r:=\operatorname{dist}\left(x_{0}, \operatorname{supp} u_{0}\right)$. We then know $R>r>0$.

We argue by contradiction. Assume that there exists $\delta>0$ such that $\operatorname{supp} u \cap\left(B_{\delta}\left(x_{0}\right) \times[0, \delta)\right)=\emptyset$. Let $\phi, 0 \leq \phi \leq 1$, be a smooth cutoff with $\operatorname{supp} \phi \subset B_{\frac{r+2 R}{3}}\left(x_{0}\right)$ and $\phi \equiv 1$ in $B_{\frac{2 r+R}{3}}\left(x_{0}\right)$.

Now obviously Lemma 16 applies to our situation. We continue to use the definition $\tau:=|\gamma|^{-\frac{29}{10}}$.

Let $\gamma_{3}<0$ be chosen such that

$$
\frac{1}{8} R^{-4}(\gamma-2)(\gamma-4+d)(-4 \gamma-2 d+8) \tau>2
$$

holds for any $\gamma<\gamma_{3}$ (such a $\gamma_{3}$ exists since $\left.\tau=|\gamma|^{-\frac{29}{10}}\right)$. 
We now prove exponential growth of the weighted entropy in case $\gamma<$ $\min \left(\gamma_{0}, \gamma_{3}\right)$ : Let $\gamma<\min \left(\gamma_{0}, \gamma_{3}\right)$. We shall show by induction that

$$
\int \sqrt{u}(x, n \tau)\left|x-x_{0}\right|^{\gamma} \phi^{4} d x \geq 2^{n} \int \sqrt{u_{0}(x)}\left|x-x_{0}\right|^{\gamma} \phi^{4} d x
$$

holds for any $n \in \mathbb{N}_{0}$ with $n \tau<\delta$. For $n=0$ this assertion is obvious. Assume that the assertion holds for $n \in \mathbb{N}_{0}$ and that $(n+1) \tau<\delta$. Then we deduce by (19) and (20) that

$$
\begin{aligned}
& \int \sqrt{u}(x,(n+1) \tau)\left|x-x_{0}\right|^{\gamma} \phi^{4} d x \\
& \geq 2 \int \sqrt{u}(x, n \tau)\left|x-x_{0}\right|^{\gamma} \phi^{4} d x \\
& \geq 2 \cdot 2^{n} \int \sqrt{u_{0}(x)}\left|x-x_{0}\right|^{\gamma} \phi^{4} d x .
\end{aligned}
$$

Thus, induction is complete.

In particular we obtain for any $T \in(0, \delta)$

$\int \sqrt{u}\left(.,|\gamma|^{-\frac{29}{10}}\left\lfloor T|\gamma|^{\frac{29}{10}}\right\rfloor\right)\left|x-x_{0}\right|^{\gamma} \phi^{4} d x \geq 2^{\left\lfloor T|\gamma|^{\frac{29}{10}}\right\rfloor} \int \sqrt{u}(., 0)\left|x-x_{0}\right|^{\gamma} \phi^{4} d x$.

Using Hölder's inequality and the fact that $\operatorname{supp} u(., t) \cap B_{\delta}(0)=\emptyset$ for $t<\delta$, we infer using $0 \leq \phi \leq 1$

$$
\begin{aligned}
\int & \sqrt{u}\left(.,|\gamma|^{-\frac{29}{10}}\left\lfloor T|\gamma|^{\frac{29}{10}}\right\rfloor\right)\left|x-x_{0}\right|^{\gamma} \phi^{4} d x \\
\leq & C(\tilde{R})\left(\int_{B_{\tilde{R}}\left(x_{0}\right)} u\left(.,|\gamma|^{-\frac{29}{10}}\left\lfloor T|\gamma|^{\frac{29}{10}}\right\rfloor\right) d x\right)^{\frac{1}{2}} \delta^{\gamma} .
\end{aligned}
$$

Moreover, using the definition (16) (recall that $M_{0}>0$ since we have $r=$ $\left.\operatorname{dist}\left(\operatorname{supp} u_{0}, x_{0}\right)\right)$ and the fact that $\phi \equiv 1$ on $\frac{B_{\frac{2 r+R}{3}}}{3}\left(x_{0}\right)$, we get

$$
\int \sqrt{u}(., 0)\left|x-x_{0}\right|^{\gamma} \phi^{4} d x \geq M_{0}\left[\frac{3 r+R}{4}\right]^{\gamma} .
$$

Putting the previous three inequalities together, we obtain

$$
C(\tilde{R})\|u\|_{L^{\infty}\left([0, \delta) ; L^{1}\left(B_{\tilde{R}}\left(x_{0}\right)\right)\right)}^{\frac{1}{2}} \delta^{\gamma} \geq 2^{\left\lfloor T|\gamma| \frac{29}{10}\right\rfloor} M_{0}\left[\frac{3 r+R}{4}\right]^{\gamma} .
$$

Letting $\gamma \rightarrow-\infty$, we now get the usual contradiction.

\section{Discussion and open problems}

We have shown that the DLSS equation displays infinite speed of support propagation; more precisely, viewing a solution $u$ of the DLSS equation as a function of both space and time, the support of $u$ has been seen to be either empty or equal to $\Omega \times[0, \infty$ ) (at least, as long $\Omega$ is connected and as long as periodic or no-flux boundary conditions are imposed). 
Unfortunately, our approach does not yield any result on the support of $u$ at any fixed time. Numerical results seem to indicate that the support of a solution of the DLSS equation is nondecreasing with respect to time [16].

However, as comparison methods are unavailable for higher-order equations and our methods do not seem to yield any stronger assertion, we do not know how to derive any such stronger result. If $d=1$ and if at a certain time $t$ the quantity $\log u$ becomes globally integrable, formal calculations suggest that it will stay integrable for all future; see e.g. [18], where in particular it is proven that $\log u_{0} \in L^{1}$ implies $\log u(., t) \in L^{1}$ for all $t>0$ for the solutions constructed in this paper. However, a localization of this result does not seem to be possible.

\section{References}

[1] Bleher, P.M., Lebowitz, J.L., Speer, E.R.: Existence and positivity of solutions of a fourth-order nonlinear pde describing interface fluctuations. Comm. Pure Appl. Math. XLVII, 923-942 (1994)

[2] Caceres, M.J., Carrillo, J.A., Toscani, G.: Long-time behaviour for a nonlinear fourth-order parabolic equation. Trans. Am. Math. Soc. 357(3), 11611175 (2004)

[3] Chen, L., Ju, Q.: Existence of weak solution and semiclassical limit for quantum drift-diffusion model. Z. Angew. Math. Phys. 58, 1-15 (2007)

[4] Chen, X., Chen, L.: The bipolar quantum drift-diffusion model. Acta Math. Sin. 25(4), 617-638 (2009)

[5] Chen, X., Chen, L., Jian, H.: The dirichlet problem of the quantum driftdiffusion model. Nonlinearity 69, 3084-3092 (2008)

[6] Chipot, M., Sideris, T.: An upper bound for the waiting time for nonlinear degenerate parabolic equations. Trans. Am. Math. Soc. 288(1), 423-427 (1985)

[7] Degond, P., Gallego, S., Mehats, F., Ringhofer, C.: Quantum Hydrodynamic models derived from the entropy principle. In: Ben Abdallah, N., Frosali, G.(eds) Quantum Transport-Modeling, Analysis, and Asymptotics, pp. 111168, Springer, Berlin (2008)

[8] Derrida, B., Lebowitz, J.L., Speer, E.R., Spohn, H.: Dynamics of an anchored Toom interface. J. Phys. A: Math. Gen. 24, 4805-4834 (1991)

[9] Dolbeault, J., Gentil, I., Jüngel, A.: A nonlinear fourth-order parabolic equation and related logarithmic Sobolev inequalities. Preprint (2004)

[10] Fischer, J.: A class of uniqueness for the Derrida-Lebowitz-Speer-Spohn equation and related quantum drift-diffusion models. Preprint (2012)

[11] Fischer, J.: Optimal lower bounds on asymptotic support propagation rates for the thin-film equation. Preprint (2012) 
[12] Fischer, J.: Upper bounds on waiting times for the thin-film equation: the case of weak slippage. Preprint (2012)

[13] Gianazza, U., Savare, G., Toscani, G.: The Wasserstein gradient flow of the Fisher information and the quantum drift-diffusion equation. Arch. Ration. Mech. Anal. 194(1), 133-220 (2009)

[14] Gualdani, M.P., Jüngel, A., Toscani, G.: A nonlinear fourth-order parabolic equation with nonhomogenous boundary conditions. SIAM J. Math. Anal. 37(6), 1761-1779 (2005)

[15] Jüngel, A., Matthes, D.: An algorithmic construction of entropies in higher-order nonlinear PDEs. Nonlinearity 19(3), 633-659 (2006)

[16] Jüngel, A.; Matthes, D: A review on results for the Derrida-Lebowitz-SpeerSpohn equation. WSPC-Proceedings (2007)

[17] Jüngel, A., Matthes, D.: The Derrida-Lebowitz-Speer-Spohn equation: existence, non-uniqueness, and decay rates of the solutions. SIAM J. Math. Anal. 39(6), 1996-2015 (2008)

[18] Jüngel, A., Pinnau, R.: Global nonnegative solutions of a nonlinear fourth-order parabolic equation for quantum systems. SIAM J. Math. Anal. 32(4), 760777 (2001)

[19] Jüngel, A., Toscani, G.: Exponential time decay of solutions to a nonlinear fourth-order parabolic equation. Z. Angew. Math. Phys. 54(3), 377-386 (2003)

[20] Jüngel, A., Violet, I.: First-order entropies for the Derrida-Lebowitz-SpeerSpohn equation. Discret. Contin. Dyn. Syst. Ser. B 8(4), 861-877 (2007)

Julian Fischer

University of Erlangen-Nuremberg

Department of Mathematics

Cauerstr. 11

91058 Erlangen

Germany

e-mail: julian.fischer@am.uni-erlangen.de

Received: 6 February 2013.

Accepted: 28 March 2013. 